\title{
Nuclear interaction gamma-ray lines from the Galactic center region
}

\author{
V. A. Dogiel ${ }^{1,2}$, V. Tatischeff ${ }^{3}$, K. S. Cheng ${ }^{4}$, D. O. Chernyshov ${ }^{1,2,4,5}$, C. M. Ko ${ }^{2}$, and W. H. Ip ${ }^{2}$
}

\author{
1 I.E. Tamm Theoretical Physics Division of P.N. Lebedev Institute, Leninskii pr, 53, 119991 Moscow, Russia \\ 2 Institute of Astronomy, National Central University, Jhongli 320, Taiwan \\ e-mail: cmko@astro.ncu.edu.tw \\ 3 Centre de Spectrométrie Nucléaire et de Spectrométrie de Masse, IN2P3-CNRS and Univ Paris-Sud, 91405 Orsay Campus, France \\ 4 Department of Physics, University of Hong Kong, Pokfulam Road, Hong Kong, PR China \\ 5 Moscow Institute of Physics and Technology, Institutskii lane, 141700 Moscow Region, Dolgoprudnii, Russia
}

Received 12 February 2009 / Accepted 1 September 2009

\section{ABSTRACT}

\begin{abstract}
Aims. The accretion of stars onto the central supermassive black hole at the center of the Milky Way is predicted to generate large fluxes of subrelativistic ions in the Galactic center region. We analyze the intensity, shape, and spatial distribution of de-excitation gamma-ray lines produced by nuclear interactions of these energetic particles with the ambient medium.

Methods. We first estimated the amount and mean kinetic energy of particles released from the central black hole during star disruption. We then calculated the energy and spatial distributions of these particles in the Galactic center region from a kinetic equation. These particle distributions were then used to derive the characteristics of the main nuclear interaction gamma-ray lines.

Results. Because the time period of star capture by the supermassive black hole is expected to be shorter than the lifetime of the ejected fast particles against Coulomb losses, the gamma-ray emission is predicted to be stationary. We find that the nuclear deexcitation lines should be emitted from a region with a maximum $5^{\circ}$ angular radius. The total gamma-ray line flux below $8 \mathrm{MeV}$ is calculated to be $\sim 10^{-4}$ photons $\mathrm{cm}^{-2} \mathrm{~s}^{-1}$. The most promising lines for detection are those at 4.44 and $\sim 6.2 \mathrm{MeV}$, with a predicted flux in each line of $\sim 10^{-5}$ photons $\mathrm{cm}^{-2} \mathrm{~s}^{-1}$. Unfortunately, it is unlikely that this emission can be detected with the INTEGRAL observatory. But the predicted line intensities appear to be within reach of future gamma-ray space instruments. A future detection of de-excitation gamma-ray lines from the Galactic center region would provide unique information on the high-energy processes induced by the central supermassive black hole and the physical conditions of the emitting region.
\end{abstract}

Key words. Galaxy: center - radiation mechanisms: non-thermal - gamma rays: theory

\section{Introduction}

Massive black holes (MBH) at galactic centers are sources of high energetic activity. X-ray observations of these sources have revealed flares of hard X-ray photons releasing a maximum power of $10^{44} \mathrm{erg} \mathrm{s}^{-1}$. These flares are supposed to caused by processes of accretion and tidal disruption of stars by the massive black holes (see, e.g., Sirota et al. 2005, and references thererin).

The capture radius of a black hole - the maximal distance from the MBH where the tidal forces can overwhelm the stellar self-gravity and tear the star apart - is given by

$R_{\mathrm{T}} \approx 1.4 \times 10^{13}\left(\frac{M_{\mathrm{bh}}}{10^{6} M_{\odot}}\right)^{1 / 3}\left(\frac{M_{*}}{M_{\odot}}\right)^{-1 / 3}\left(\frac{R_{*}}{R_{\odot}}\right) \mathrm{cm}$,

where $M_{*}$ and $R_{*}$ are the star's mass and radius, $M_{\mathrm{bh}}$ the mass of the black hole, and $M_{\odot}$ and $R_{\odot}$ the solar mass and radius. When a star comes within the capture radius of a black hole, the tidal force produced by the black hole is strong enough to capture the star (Rees 1988; Phinney 1989). If the mass of the black hole is higher than $\sim 10^{8} M_{\odot}$, the star directly falls into the black hole event horizon, and not many interesting phenomena can be observed. However, if the mass of the black hole is less than $\sim 10^{8} M_{\odot}$, the star is torn apart. Roughly half of the star is captured by the black hole and the disrupted debris forms a circular transient accretion disk around the black hole in a few months (Ulmer 1999). The time-dependent accretion rate follows a simple power law, $\mathrm{d} M / \mathrm{d} t \propto t^{-5 / 3}$, with an initial accretion higher than the Eddington rate.

Total tidal disruption of a star occurs when the penetration parameter $b^{-1} \gg 1$, where $b$ is the ratio of $r_{\mathrm{p}}$, the periapse distance (distance of closest approach) to the tidal radius $R_{\mathrm{T}}$. The tidal disruption rate $v_{\mathrm{s}}$ can be approximated to within an order of magnitude from an analysis of star dynamics near a black hole via the Fokker-Planck equation. For the parameters of the GC, it gives the rate $v_{\mathrm{s}} \sim 10^{-4}$ years $^{-1}$ (see the review of Alexander 2005), which agrees with the more detailed calculations of Syer \& Ulmer (1999), who obtained $v_{\mathrm{s}} \sim 5 \times 10^{-5}$ years $^{-1}$. Tidal disruption processes have perhaps already been observed in cosmological galaxy surveys (see, e.g. Donley et al. 2002).

As observations show, there is a supermassive black hole $\left(\operatorname{Sgr} A^{*}\right)$ in the center of our Galaxy with a mass of $(4.31 \pm$ $0.06) \times 10^{6} M_{\odot}($ Gillessen et al. 2008). In the close vicinity of the Galactic black hole (less than 0.04 pc from it), about 35 low-mass stars $\left(1-3 M_{\odot}\right)$ and about 10 massive stars (3-15 $\left.M_{\odot}\right)$ are present (see Alexander \& Livio 2004); i.e., processes of star accretion in the Galactic center seems to be quite possible. However, attempts to find a strong X-ray source in the Galactic center failed. The Chandra X-ray Observatory only resolved a weak X-ray point source at the position of Sgr A* with a flux 
$L_{\mathrm{X}} \sim 10^{33} \mathrm{erg} \mathrm{s}^{-1}$ (see Baganoff et al. 2003), though moderate X-ray flares were observed by Chandra (Porquet et al. 2008). As mentioned by Koyama et al. (1996) and Muno et al. (2004), this "X-ray quiet" Sgr A* sharply contrasts to the high X-ray activity of the surrounding diffuse hot plasmas. One likely scenario is that the Galactic nucleus was brighter in the past, possibly caused by surge accretion onto the massive back hole.

Cheng et al. $(2006,2007)$ suggested that this scenario may explain the origin of the $511 \mathrm{keV}$ annihilation flux from the GC region, if up to $10 \%$ of captured stellar matter is ejected in the form of a jet of relativistic protons. In this case the origin of the $511 \mathrm{keV}$ line emission from the GC region is supposed to caused by annihilation of secondary positrons generated by $\mathrm{p}-\mathrm{p}$ collisions.

An attempt to find independent evidence of active processes in the GC in the form of a flux of fast charged particles was undertaken by Dogiel et al. (2008), who conclude that these primary relativistic protons (if generated) penetrating the dense molecular clouds produced a flux there of nuclear de-excitation gamma-ray lines coming from the GC region. However, the origin of relativistic protons is still rather speculative since we do not have direct evidence of their production near black holes (see, however, Abraham et al. 2007; Istomin \& Sol 2009). Besides, the gamma-ray line flux would be strongly time variable in this case, making the estimates of its value at present are highly model-dependent.

Below we examine a different model of gamma-ray line emission, assuming nuclear interactions of subrelativistic protons generated by processes of star disruption at the GC.

\section{Flux of subrelativistic protons generated by star disruption}

An alternative (and to our view more reliable) mechanism of gamma-ray line production follows from the analysis of processes of star disruption near black holes. The energy budget of a tidal disruption event follows from analysis of star matter dynamics. Once passing the pericenter, the star is tidally disrupted into a very long and dilute gas stream. The outcome of tidal disruption is that some energy is extracted out of the orbit to unbind the star and accelerate the debris. Initially about $50 \%$ of the stellar mass becomes tightly bound to the black hole, while the remainder $50 \%$ of the stellar mass is forcefully ejected (see, e.g. Ayal et al. 2000). The kinetic energy carried by the ejected debris is a function of the penetration parameter $b^{-1}$ that can significantly exceed that released by a normal supernova $\left(\sim 10^{51} \mathrm{erg}\right)$ if the orbit is highly penetrating (see Alexander 2005),

$W \sim 4 \times 10^{52}\left(\frac{M_{*}}{M_{\odot}}\right)^{2}\left(\frac{R_{*}}{R_{\odot}}\right)^{-1}\left(\frac{M_{\mathrm{bh}} / M_{*}}{10^{6}}\right)^{1 / 3}\left(\frac{b}{0.1}\right)^{-2} \mathrm{erg}$.

Thus, the mean kinetic energy per escaping nucleon is given by

$E_{\mathrm{esc}} \sim 42\left(\frac{\eta}{0.5}\right)^{-1}\left(\frac{M_{*}}{M_{\odot}}\right)\left(\frac{R_{*}}{R_{\odot}}\right)^{-1}\left(\frac{M_{\mathrm{bh}} / M_{*}}{10^{6}}\right)^{1 / 3}\left(\frac{b}{0.1}\right)^{-2} \mathrm{MeV}$,

where $\eta M_{*}$ is the mass of escaping material. For the black-hole mass $M_{\mathrm{bh}}=4.31 \times 10^{6} M_{\odot}$, the energy of escaping particles is $E_{\text {esc }} \sim 68(\eta / 0.5)^{-1}(b / 0.1)^{-2} \mathrm{MeV}$ nucleon $^{-1}$ when a one-solar mass star is captured. The parameters $\eta$ and $b$ are not wellconstrained by theory. It is clear, however, that $b$ must be less than unity for a star to be disrupted in the gravitational potential of the black hole. We take the energy distribution of the erupted nuclei as a simple Gaussian distribution

$Q_{0}^{\mathrm{esc}}=\frac{N}{\sigma \sqrt{2 \pi}} \exp \left[-\frac{\left(E-E_{\mathrm{esc}}\right)^{2}}{2 \sigma^{2}}\right]$,

where $N$ is total amount of particles ejected by one stellar capture. Results of calculations for different values of $\sigma$ do not differ much from each other as long as $\sigma \leq E_{\text {esc }}$ (see below).

For a single capture of a one-solar mass star, the total number of unbounded particles is $N \lesssim 10^{57}$, and the total kinetic energy in these particles is $W \lesssim 10^{53} \mathrm{erg}$, if their energy is about $68 \mathrm{MeV}$ (see above). For the star capture frequency $v_{\mathrm{s}} \simeq 10^{-4}$ year $^{-1}$ and $E_{\text {esc }}$ about several ten MeV (see, Dogiel et al. 2009b) it gives a power input $\dot{W} \lesssim 3 \times 10^{41} \mathrm{erg} \mathrm{s}^{-1}$, which is a few times more than the total power contained in Galactic cosmic rays. The average energy output depends, of course, on the capture frequency.

A capture of a massive star should eject more fast particles into the GC region than a capture of a low-mass star. However, the energy of the ejected particles is expected to be similar. This can be seen from Eq. (3), recalling that the radius of mainsequence stars varies as $R_{*} \propto M_{*}^{0.8}$ in first approximation. The frequency of massive star capture is lower than for low-mass stars (see, e.g. Syer \& Ulmer 1999). Therefore, we expect the capture of a massive star to have relatively little effect in comparison with the cumulative effect from low-mass star captures. The latter is calculated below assuming $M_{*}=1 M_{\odot}$ for all captured stars.

\section{Proton propagation in the Galactic center region}

Details of the proton spatial distribution in the GC region, as well as the mechanism of proton propagation are inessential for calculations of the total gamma-ray line flux. We use, nevertheless, kinetic equations in which processes of propagation, are included, and then integrate the obtained solution over the volume of emission. The effect of propagation reduces in this case to a proper estimate of the proton flux leaving the emission region.

Propagation of cosmic rays in the Galaxy is described as a diffusion phenomenon, with a phenomenological diffusion coefficient whose value and energy dependence is derived from observational data, e.g., from the chemical composition of cosmic rays measured near Earth. This method leads to a diffusion coefficient of about $10^{27} \mathrm{~cm}^{2} \mathrm{~s}^{-1}$ (for details see Berezinskii et al. 1990). Processes of particle propagation in the GC region are questionable since we do not know much about the physical conditions of the ambient medium.

From the general theory of cosmic ray origin, it is clear that particle propagation in the interstellar medium is described as diffusion due to scattering on magnetic fluctuations. The effective coefficient of diffusion is $D \sim v^{2} / v(E, \boldsymbol{r})$, where $v$ is the particle velocity and $v(E, \boldsymbol{r})$ is the scattering frequency of particles, which is a function of particle energy $E$ and coordinates $r$. Fairly simple estimates provided by Jean et al. (2006) for the GC region in the frame of the quasi-linear theory gives $D \sim 10^{27} \mathrm{~cm}^{2} \mathrm{~s}^{-1}$ for $\mathrm{MeV}$ particles. A similar estimate was obtained by LaRosa et al. (2001) from analysis of radio emission from the central region. These authors conclude that electrons of $0.14 \mathrm{GeV}$ diffuse about $13 \mathrm{pc}$ in $4 \times 10^{4}$ years, which again gives $D \sim 10^{27} \mathrm{~cm}^{2} \mathrm{~s}^{-1}$.

Estimates of the diffusion coefficient for subrelativistic protons in the GC have been derived by Dogiel et al. (2009c) from the observed hard X-ray emission, which is assumed to be due to inverse bremsstrahlung. They estimated $D$ to be in the range 
$10^{26}-10^{27} \mathrm{~cm}^{2} \mathrm{~s}^{-1}$. Below we provide our calculations for the diffusion coefficient $D=10^{27} \mathrm{~cm}^{2} \mathrm{~s}^{-1}$, though, in principle, its value may be a function of particle energy (as well as of spatial coordinates and even time). We suppose, however, that this simplification is acceptable because the energy range of relevance for gamma-ray line production is quite narrow, less than one order of magnitude.

Another important parameter of the model is the mean density of the medium into which the fast nuclei propagate. Observations show that the interstellar medium near the GC is highly nonuniform. Thus, within the central $2 \mathrm{pc}$ of the shell of the supernova remnant Sgr A East, there is a hot plasma of temperature $2 \mathrm{keV}$ and density as high as $10^{3} \mathrm{~cm}^{-3}$ (Maeda et al. 2002). This local density fluctuation is insignificant for our treatments since energetic particles capable of producing de-excitation gamma-ray lines lose little energy passing through this local fluctuation by diffusion.

A significant part of the gas in the GC region is in the form of molecular hydrogen, $\sim 90 \%$ of which is contained in dense and massive molecular clouds with a volume-filling factor of only a few per cent. The total mass of molecular hydrogen in the nuclear bulge, which is estimated to be $2 \times 10^{7} M_{\odot}$, significantly exceeds the mass contained in the intercloud hot plasma (see Launhardt et al. 2002).

However, subrelativistic charged particles may not be able to penetrate freely inside molecular clouds (see, e.g., Skilling $\&$ Strong 1976). Theoretical investigations performed by Dogiel et al. $(1987,2005)$ showed that turbulent motions of neutral gas could excite small-scale electromagnetic fluctuations that could prevent charged particles to penetrate deeply into clouds. Thus, subrelativistic cosmic-ray particles are expected to fill only a small portion of molecular clouds. This conclusion is supported by calculations performed by Dogiel et al. (2009a), who show that the flux of bremsstrahlung emission produced by subrelativistic protons inside molecular clouds is lower than produced in the intercloud hot plasma. If this is true, we expect that most of the gamma-ray line emission should also be generated in the intercloud hot plasma.

Recent observations with the satellites ASCA, Chandra, and $S u z a k u$ have shown that the $1-2^{\circ}$ radius central region is filled with a hot gas of temperature $6-10 \mathrm{keV}$. The density of plasma derived from these observations ranges between $n \simeq 0.1-0.4 \mathrm{~cm}^{-3}$ (Koyama et al. 1996; Muno et al. 2004; Koyama et al. 2007). In the calculations we use the average value $n=0.2 \mathrm{~cm}^{-3}$. For these parameters the average time of Coulomb losses for 10-100 MeV protons is (see, e.g. Hayakawa 1969)

$\tau_{i} \sim \frac{E_{\mathrm{p}} m_{\mathrm{e}} v_{\mathrm{p}}}{6 \pi n e^{4} \ln \Lambda} \sim 3 \times 10^{6}-3 \times 10^{7} \mathrm{yr}$,

where $E_{\mathrm{p}}$ and $v_{\mathrm{p}}$ are the proton energy and velocity, and $\ln \Lambda$ is the Coulomb logarithm.

\section{Spatial and energy distributions of subrelativistic protons in the Galactic center region}

The time-dependent spectrum of subrelativistic protons erupted from the central region can be calculated from the equation

$$
\frac{\partial f}{\partial t}-\nabla(D \nabla f)+\frac{\partial}{\partial E}\left(\frac{\mathrm{d} E}{\mathrm{~d} t} f\right)=Q(E, t)
$$

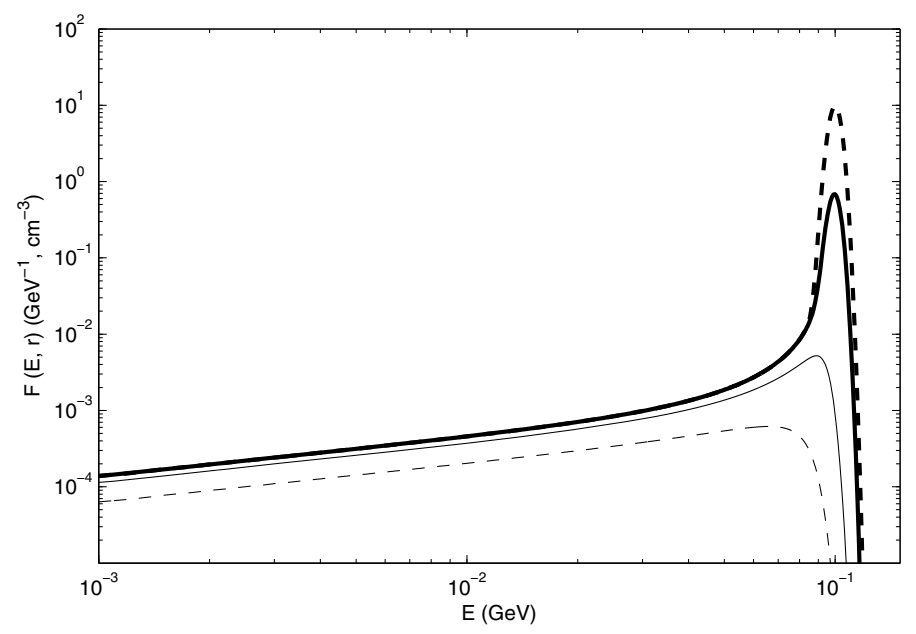

Fig. 1. Energy spectra of primary protons at $r=0 \mathrm{pc}$ and $t=10^{3}$ years (thick dashed line), $r=0 \mathrm{pc}$ and $t=10^{4}$ years (thick solid line), $r=150 \mathrm{pc}$ and $t=10^{4}$ years (thin solid line), and $r=300 \mathrm{pc}$, $t=10^{4}$ years (thin dashed line). For $r>10 \mathrm{pc}$ the proton spectra are almost stationary. These spectra were calculated for $E_{\mathrm{esc}}=100 \mathrm{MeV}$ and $\sigma=0.05 E_{\text {esc }}$.

where $\mathrm{d} E / \mathrm{d} t \equiv b(E)$ is the rate of proton energy losses. Subrelativistic protons lose their energy by Coulomb collisions. The rate of losses for these protons can be presented in the form

$b(E)=-\frac{2 \pi n e^{4}}{m_{e} \mathrm{~V}} \ln \Lambda \simeq-\frac{a}{\sqrt{E}}$.

The injection of protons by processes of star capture can be described by

$Q(E, \boldsymbol{r}, t)=\sum_{k=0} Q_{k}(E) \delta\left(t-t_{k}\right) \delta(\boldsymbol{r})$,

where $t_{k}=k \times T$ is the injection time and the functions $Q_{k}(E)$ are given by Eq. (4). The mean time of star capture by the massive black hole in the Galaxy is taken to be $T \simeq 10^{4}$ years.

We can derive the Green function of Eq. (6) for the injection time $t_{k}$ (see Syrovatskii 1959; Gratton 1972) and then sum over injections. The equation for the Green function is

$\frac{\partial G_{k}}{\partial t}-\nabla\left(D \nabla G_{k}\right)+\frac{\partial}{\partial E}\left(b(E) G_{k}\right)=\delta\left(E-E_{0}\right) \delta\left(t-t_{k}\right) \delta(\boldsymbol{r})$.

Using variables

$\tau\left(E, E_{0}\right)=\int_{E_{0}}^{E} \frac{\mathrm{d} E}{b(E)} \quad$ and $\quad \lambda=\int_{E_{0}}^{E} \frac{D(E)}{b(E)} \mathrm{d} E$,

the Green function can be written as

$G_{k}\left(\boldsymbol{r}, E, t ; E_{0}, t_{k}\right)=\frac{1}{|b(E)|} \frac{\delta\left(t-t_{k}-\tau\right)}{(4 \pi \lambda)^{3 / 2}} \exp \left[-\frac{\boldsymbol{r}^{2}}{4 \lambda}\right]$.

Then, $f(\boldsymbol{r}, E, t)$ is

$f(\boldsymbol{r}, E, t)=\sum_{k=0} \int_{0}^{\infty} \mathrm{d} E_{0} Q_{k}\left(E_{0}\right) G_{k}\left(\boldsymbol{r}, E, t ; E_{0}, t_{k}\right)$.

In the nonrelativistic case, i.e. for energy losses in the form of Eq. (7), the solution (12) can be simplified to

$f(\boldsymbol{r}, E, t)=\sum_{k=0} \frac{N_{k} \sqrt{E}}{\sigma \sqrt{2 \pi} Y_{k}^{1 / 3}} \frac{\exp \left[-\frac{\left(E_{\mathrm{esc}}-Y_{k}^{2 / 3}\right)^{2}}{2 \sigma^{2}}-\frac{r^{2}}{4 D\left(t-t_{k}\right)}\right]}{\left(4 \pi D\left(t-t_{k}\right)\right)^{3 / 2}}$, 


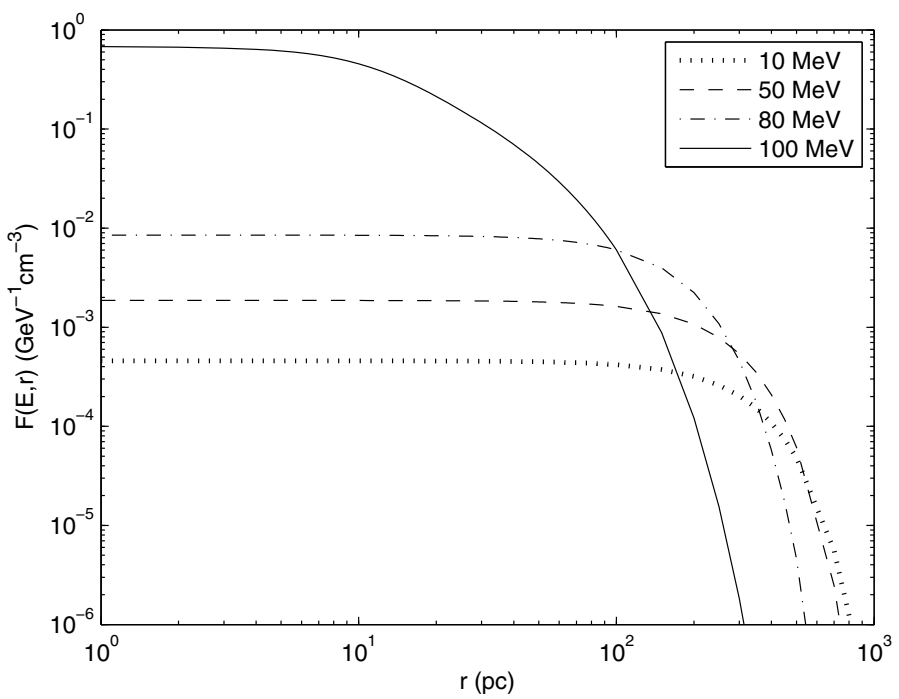

Fig. 2. Densities in protons of energies $10,50,80$, and $100 \mathrm{MeV}$, as a function of radial distance from the GC. These spatial distributions were calculated for $E_{\mathrm{esc}}=100 \mathrm{MeV}, \sigma=0.05 E_{\mathrm{esc}}$, and $n=0.2 \mathrm{~cm}^{-3}$.

where

$Y_{k}(t, E)=\left[\frac{3 a}{2}\left(t-t_{k}\right)+E^{3 / 2}\right]$.

As one can see, this solution is characterized by two different spatial scales. The first one is $R_{1} \sim \sqrt{4 D T} \sim 10 \mathrm{pc}$. Inside the sphere $r<R_{1}$, the solution is time dependent and the density of protons fluctuates strongly with time. On the other hand, for $r>R_{1}$ the solution is quasi-stationary because the time of diffusion over these distances is longer than the mean capture time $T$. The radius of the sphere filled with subrelativistic protons (the scale of exponential decrease) is about $R_{2} \sim \sqrt{4 D \tau_{i}} \sim 200 \mathrm{pc}$ for $E_{\mathrm{esc}}=100 \mathrm{MeV}$. One can see from Eq. (5) that the mean free path of protons is proportional to $\sqrt{1 / n}$. Examples of calculated energy and spatial distributions of subrelativistic protons are shown in Figs. 1 and 2.

Since the Galactic black hole was inactive for a long time, we calculated the gamma-ray line emission the proton spectrum at time $t=T$ after the last star capture. This will give us a lower limit on the gamma-ray line flux. However, the density of subrelativistic protons in the $\mathrm{GC}$ is expected to be almost independent of time, except for a very compact region around the massive black hole. The proton spectrum should reach its saturation level after $\gtrsim \tau_{i} / T$ capture events. In our calculations we summarized the cumulative effect of 2000 captures.

To predict the total gamma-ray line flux from the GC we need to estimate the total (integrated over volume) spectrum of subrelativistic protons

$F(E, t)=4 \pi \int_{0}^{\infty} f(\boldsymbol{r}, E, t) r^{2} \mathrm{~d} r$.

We note that for simple estimates one can derive this spectrum from the assumption of a stationary injection of protons with the rate $Q=N / T$. Then the approximate solution has the form

$\bar{F}(E)=\frac{Q \sqrt{E}}{2 a}\left[1+\operatorname{Erf}\left(\frac{E_{\mathrm{esc}}-E}{\sqrt{2} \sigma}\right)\right]$

where $\operatorname{Erf}(x)$ is the error function.

\section{Nuclear interaction gamma-ray line emission from the Galactic center region}

Unlike relativistic particles, subrelativistic nuclei do not effectively produce continuous radiation (except, may be, inverse bremsstrahlung, which is discussed in Dogiel et al. 2009a,b,c). However, collisions of subrelativistic nuclei with ambient matter can lead to nuclear excitation and emission of de-excitation gamma-ray lines. These lines may be a good tracer for subrelativistic cosmic rays, because the line brightness can give us information about the amount of subrelativistic particles.

Thus, prominent nuclear de-excitation lines are observed in emission spectra of solar flares, which allows derivation of valuable information on solar ambient abundances, density and temperature, as well as on accelerated particle composition, spectra, and transport in the solar atmosphere (see e.g., Smith et al. 2003; and Kiener et al. 2006, for recent solar observations with RHESSI and INTEGRAL, respectively). Dogiel (2001) predicted that galaxy clusters could emit a detectable flux of de-excitation gamma-ray lines. Later, Iyudin et al. (2004) found traces of gamma-ray line emission towards the Coma and Virgo clusters, though this detection has not been confirmed yet.

Detection of the gamma-ray line emission produced by cosmic-ray interactions in the interstellar medium would provide insightful information on low-energy cosmic rays and give a significant advance in the development of the theory of cosmic-ray origin. Theoretical estimates for the line emission in the Galaxy were provided by Ramaty et al. (1979), who calculated spectra of the nuclear de-excitation line emission for different assumed spectra of subrelativistic ions. However, attempts to measure a prominent flux of nuclear interaction lines were failed up to now.

Galactic center observations in the $\mathrm{MeV}$ range were performed by the COMPTEL group but with a rather poor angular resolution (Bloemen et al. 1997). They detected a marginal excess of emission in the range 3-7 MeV. The total flux from the central region $60^{\circ} \times 20^{\circ}$ was estimated to be $10^{-4} \mathrm{~cm}^{-2} \mathrm{~s}^{-1} \mathrm{rad}^{-1}$. This excess was interpreted as emission in nuclear de-excitation lines generated by cosmic-ray interaction in the GC region. However, neither the COMPTEL group nor subsequent observations with INTEGRAL have confirmed this result. In particular, Teegarden \& Watanabe (2006) carried out an extensive search for gamma-ray lines in the first year of public data from the INTEGRAL spectrometer SPI and found no evidence of any previously unknown lines in the energy range $20-8000 \mathrm{keV}$.

We suppose that processes of star accretion by the massive black hole at the GC can generate a significant number of subrelativistic protons and heavier nuclei. These energetic particles could in turn produce a significant flux of nuclear deexcitation gamma-ray lines in a relatively compact region of the GC. Unlike the gamma-ray emission that might be produced by relativistic protons (Dogiel et al. 2008), the emission produced by subrelativistic protons is expected to be almost stationary, so should be less dependent on model parameters.

Though the origin of subrelativistic cosmic rays near the GC is different than in other part of the galactic disk according to the present model, a positive detection of de-excitation gammaray lines would provide important information about star accretion processes at the GC. We can calculate the gamma-ray flux emitted at time $t=T$ after a star capture in a given nuclear deexcitation line from

$F_{\gamma}(t)=\frac{n_{\mathrm{H}}}{4 \pi d^{2}} \sum_{i j} \frac{n_{j}}{n_{\mathrm{H}}} \int_{0}^{\infty} F_{i}\left(E_{i}, t\right) v_{i}\left(E_{i}\right) \sigma_{i j}\left(E_{i}\right) \mathrm{d} E_{i}$, 


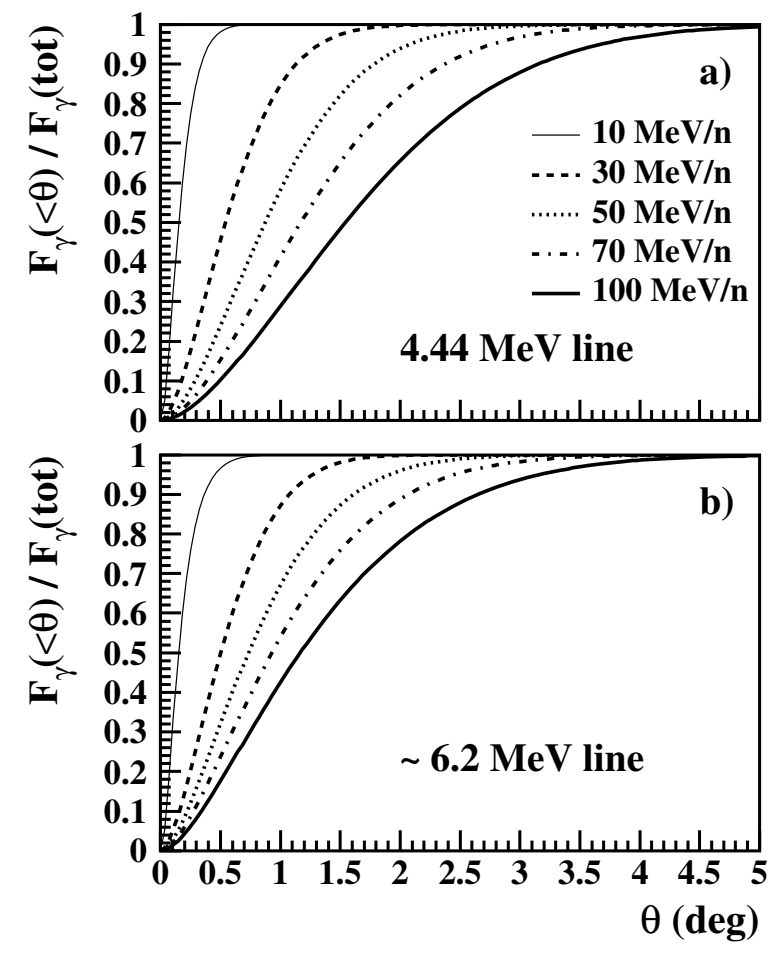

Fig. 3. Radial profiles of enclosed fluxes in a) the $4.44 \mathrm{MeV}$ line and b) the $\sim 6.2 \mathrm{MeV}$ line complex, as a function of angular radius from the $\mathrm{GC}$, for the five values of $E_{\mathrm{esc}}$ indicated in the figure. The calculations assume $\sigma=0.05 E_{\text {esc }}$ (Eq. (4)) and $n_{\mathrm{H}}=0.2 \mathrm{~cm}^{-3}$.

where $i$ and $j$ range over the accelerated and ambient particle species that contribute to the production of the gamma-ray line considered, $n_{\mathrm{H}}=0.2 \mathrm{~cm}^{-3}$ is the adopted mean density of $\mathrm{H}$ atoms in the interaction region, $n_{j}$ the density of the ambient constituent $j, F_{i}\left(E_{i}, t\right)$ the energy spectrum at time $t$ of the fast particles in the interaction region (Eq. (15)), $v_{i}$ the velocity of these particles, $\sigma_{i j}$ the cross section for the reaction of interest between species $i$ and $j$, and $d=8 \mathrm{kpc}$ the distance to the GC (Grönewegen et al. 2008). To take the measured enhancement of the metal abundances in the GC region into account (Cunha et al. 2007), we assumed the abundances of ambient carbon and heavier elements to be twice solar (Lodders 2003). This is consistent with the recent estimate from Suzaku observations of Koyama et al. (2009), who found the Fe abundance to be between 1.5 and 2.3 times solar. In the case of a low-mass star capture, we expect the line emission to mainly stem from proton and $\alpha$-particle reactions with ambient heavy nuclei. We took the $\alpha$ particles into account assuming, for simplicity, $F_{\alpha}(E, t)=X_{\alpha} F_{\mathrm{p}}(E, t)$, where the energy $E$ is expressed in $\mathrm{MeV}$ nucleon ${ }^{-1}$ and $X_{\alpha}=0.1$ is the accelerated $\alpha / \mathrm{p}$ abundance ratio.

Noteworthy is that the total steady-state emission obtained from Eq. (17) independent of the assumed mean density in the interaction region, as can be seen by introducing in this equation the particle distribution $F_{i}(E, t)$, which is inversely proportional to $n_{\mathrm{H}}$ (see, e.g., Eq. (16)). This is true, however, as long as $n_{\mathrm{H}} \lesssim$ $100 \mathrm{~cm}^{-3}$, because for higher density values the Coulomb loss time $\tau_{i}<T$ and the particle distribution is no longer stationary. In any case, even if the predicted emission were time dependent, the total fluxes given below can be interpreted as mean values over the period of star capture $T$.

Figure 3 shows calculated radial profiles of the predicted line emission at 4.44 and $\sim 6.2 \mathrm{MeV}$. These two lines are mainly produced by proton and $\alpha$-particle reactions with ambient ${ }^{12} \mathrm{C}$ and
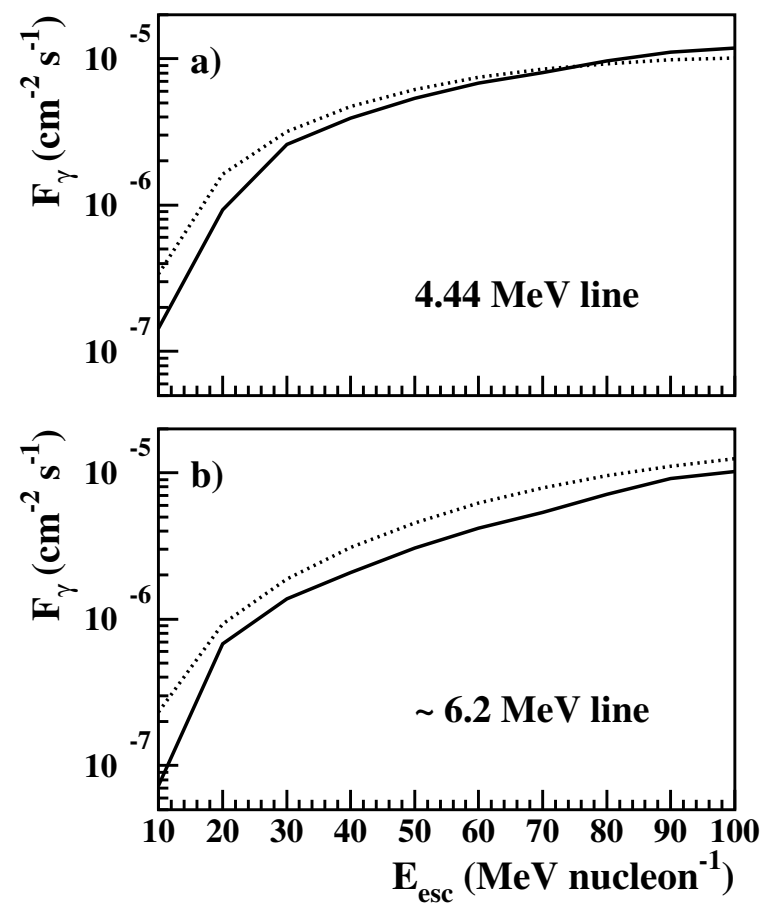

Fig. 4. Predicted fluxes from the GC region of angular radius $\theta=5^{\circ}$ in a) the $4.44 \mathrm{MeV}$ line and b) the $\sim 6.2 \mathrm{MeV}$ line complex, as a function of $E_{\mathrm{esc}}$, for $\sigma=0.05 E_{\mathrm{esc}}$ (solid lines), and $\sigma=E_{\mathrm{esc}}$ (dashed lines).

${ }^{16} \mathrm{O}$ (see below). In contrast to the total emission from the GC region (i.e. integrated over space), the radial profiles depend on the assumed value of $n_{\mathrm{H}}$ : the higher the ambient medium density, the lower the spatial extend of the gamma-ray line emission. We see in Fig. 3 that, for $n_{\mathrm{H}}=0.2 \mathrm{~cm}^{-3}$ and $E_{\mathrm{esc}} \leq 100 \mathrm{MeV}$ nucleon ${ }^{-1}$, the gamma-ray lines are emitted from a region of maximum $5^{\circ}$ angular radius. Such emission would appear as small-scale diffuse emission for a gamma-ray instrument like SPI, whose angular resolution is $\sim 3^{\circ} \mathrm{FWHM}$ (see, e.g., Teegarden \& Watanabe 2006).

Figure 4 shows calculated fluxes in the 4.44 and $\sim 6.2 \mathrm{MeV}$ lines as a function of $E_{\mathrm{esc}}$ ranging from 10 to $100 \mathrm{MeV}$ nucleon $^{-1}$, which corresponds to $0.68<$ $(\eta / 0.5)(b / 0.1)^{2}<6.8$ (see Eq. (3)). We see that the predicted fluxes increase with increasing escape energy up to values $\approx 10^{-5}$ photons $\mathrm{cm}^{-2} \mathrm{~s}^{-1}$ for $E_{\mathrm{esc}} \approx 100 \mathrm{MeV}$ nucleon $^{-1}$. We also see that the fluxes are not strongly dependent on the assumed width of the adopted Gaussian distribution ( $\sigma$ in Eq. (4)).

Dogiel et al. (2009b) show that the hard X-rays observed with Suzaku from the GC region (Yuasa et al. 2008) can be produced by inverse bremsstrahlung from subrelativistic protons accelerated by star disruption near Sgr $\mathrm{A}^{*}$. In this scenario, the measured spectrum of this hard X-ray emission would imply that $E_{\mathrm{esc}} \approx 100 \mathrm{MeV}$ nucleon $^{-1}$. Figure 5 shows a gamma-ray line spectrum calculated for $E_{\mathrm{esc}}=100 \mathrm{MeV}$ nucleon $^{-1}$ and $\sigma=0.05 E_{\text {esc }}$, using the code developed by Ramaty et al. (1979). The calculated total flux in gamma-ray lines below $8 \mathrm{MeV}$ is $1.1 \times 10^{-4}$ photons $\mathrm{cm}^{-2} \mathrm{~s}^{-1}$. In the $3-7 \mathrm{MeV}$ range, it is $4.4 \times 10^{-5}$ photons $\mathrm{cm}^{-2} \mathrm{~s}^{-1}$.

We see relatively strong lines of various widths in Fig. 5, which are superimposed on a continuum-like emission due to all the unresolved gamma-ray lines, mostly arising from cascade transitions in high-lying levels of heavy nuclei. Among the strongest lines are those produced in excitations of low-lying nuclear levels of abundant ambient ions, e.g. at $4.44 \mathrm{MeV}$ from 


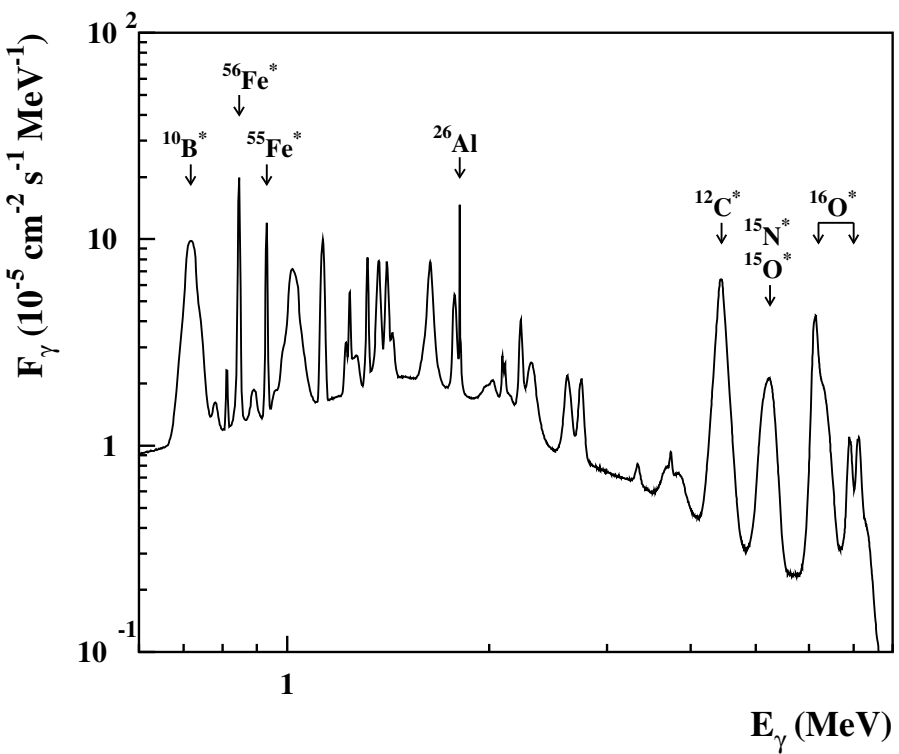

Fig. 5. Spectrum of the predicted gamma-ray line emission from the GC region of angular radius $\theta=5^{\circ}$, assuming $E_{\mathrm{esc}}=100 \mathrm{MeV}$ nucleon $^{-1}$ and $\sigma=0.05 E_{\text {esc }}$. The arrows point to the lines that are mentioned in the text.

${ }^{12} \mathrm{C}^{*}$ and $0.847 \mathrm{MeV}$ from ${ }^{56} \mathrm{Fe}^{*}$. Other important lines are those arising from transitions in the spallation products of abundant ambient nuclei, e.g. at $0.718 \mathrm{MeV}$ from ${ }^{10} \mathrm{~B}^{*}$, which is produced by spallation of ambient ${ }^{12} \mathrm{C}$ and ${ }^{16} \mathrm{O}$, at $0.931 \mathrm{MeV}$ from ${ }^{55} \mathrm{Fe}^{*}$, which is mainly produced by the reaction ${ }^{56} \mathrm{Fe}(\mathrm{p}, \mathrm{pn}){ }^{55} \mathrm{Fe}^{*}$ and at $\sim 5.2 \mathrm{MeV}$ from ${ }^{15} \mathrm{~N}^{*}$ and ${ }^{15} \mathrm{O}^{*}$ resulting from spallation of ${ }^{16} \mathrm{O}$ (see Kozlovsky et al. 2002). The broad line feature at $\sim 6.2 \mathrm{MeV}$ comes from the merging of the $6.129 \mathrm{MeV}$ line from de-excitations in ${ }^{16} \mathrm{O}^{*}$ with two lines at $6.175 \mathrm{MeV}$ from ${ }^{15} \mathrm{O}^{*}$ and $6.322 \mathrm{MeV}$ from ${ }^{15} \mathrm{~N}^{*}$ produced in spallation reactions. The characteristics of the main gamma-ray lines are summarized in Table 1.

The lines produced in a gaseous ambient medium are broadened by the recoil velocity of the excited nucleus. The line width generally decreases with increasing mass of the target atoms, because the recoil velocity of heavier nuclei is lower. Thus, in Fig. 5, FWHM (of the $0.847 \mathrm{MeV}$ line produced by proton and $\alpha$-particle inelastic scattering of ${ }^{56} \mathrm{Fe}$ is $6 \mathrm{keV}(0.7 \%$ of the transition energy), whereas the $F W H M$ of the $4.44 \mathrm{MeV}$ from ${ }^{12} \mathrm{C}^{*}$ is $160 \mathrm{keV}\left(\Delta E_{\gamma} / E_{\gamma}=3.6 \%\right)$. However, some gamma-ray lines produced in interstellar dust grains can be very narrow, because some of the excited nuclei can stop in solid materials before emitting gamma rays (Lingenfelter \& Ramaty 1977). It requires that the mean lifetime of the excited nuclear level or of its radioactive nuclear parent is longer than the slowing down time of the excited nucleus in the grain material. The most promising of such lines is that at $6.129 \mathrm{MeV}$ from ${ }^{16} \mathrm{O}^{*}$. Thus, future measurements of the profile of the $\sim 6.2 \mathrm{MeV}$ line could allow us to probe for the presence of dust grains in the GC region and perhaps to measure the grain size distribution (Tatischeff \& Kiener 2004).

Very narrow lines can also result from the decay of spallation-produced long-lived radionuclei, which can come to rest essentially in the ambient medium before decaying to excited states of their daughter nuclei and emitting gamma rays. For $n_{\mathrm{H}} \approx 0.2 \mathrm{~cm}^{-3}$, the slowing-down time of recoiling heavy nuclei excited by fast proton and $\alpha$-particle collisions is around $10^{4}$ years. Thus, only radionuclei with mean lifetimes longer
Table 1. Main gamma-ray lines expected from the GC region

\begin{tabular}{|c|c|c|c|}
\hline $\begin{array}{l}\text { Line energy } \\
(\mathrm{MeV})\end{array}$ & $\begin{array}{l}\text { Nuclear } \\
\text { Transition }\end{array}$ & $\begin{array}{c}F W H M^{a} \\
(\mathrm{keV})\end{array}$ & $\begin{array}{c}\begin{array}{c}\text { Flux }^{a} \\
\mathrm{~cm}^{-2} \mathrm{~s}^{-1}\end{array} \\
\end{array}$ \\
\hline 0.718 & ${ }^{10} \mathrm{~B}_{0.718}^{*} \rightarrow$ g.s. ${ }^{b}$ & 30 & $3.4 \times 10^{-6}$ \\
\hline 0.847 & $\begin{array}{l}{ }^{56} \mathrm{Fe}_{0.847}^{*} \rightarrow \text { g.s. } \\
{ }^{27} \mathrm{Al}_{084}^{*} \rightarrow \text { g.s. }\end{array}$ & 6 & $1.4 \times 10^{-6}$ \\
\hline 1.809 & $\begin{array}{c}{ }^{26} \mathrm{Mg}_{1.809}^{*} \rightarrow \text { g.s. } \\
\left({ }^{26} \mathrm{Al} \text { decay }\right)\end{array}$ & $<2.7^{c}$ & $3.5 \times 10^{-7}$ \\
\hline 4.44 & $\begin{array}{l}{ }^{12} \mathrm{C}_{4.439}^{*} \rightarrow \text { g.s. } \\
{ }^{11} \mathrm{~B}_{4.445}^{*} \rightarrow \text { g.s. }\end{array}$ & 160 & $1.2 \times 10^{-5}$ \\
\hline$\sim 5.2$ & $\begin{array}{l}{ }^{14} \mathrm{~N}_{5.106}^{4.445} \rightarrow \text { g.s. } \\
{ }^{15} \mathrm{O}_{5.181}^{*} \rightarrow \text { g.s. } \\
{ }^{15} \mathrm{O}_{5.241}^{*} \rightarrow \text { g.s. } \\
{ }^{15} \mathrm{~N}_{5.270}^{*} \rightarrow \text { g.s. } \\
{ }^{15} \mathrm{~N}_{5.299}^{*} \rightarrow \text { g.s. }\end{array}$ & 280 & $5.4 \times 10^{-6}$ \\
\hline$\sim 6.2$ & $\begin{array}{l}{ }^{16} \mathrm{O}_{6.130}^{*} \rightarrow \text { g.s. } \\
{ }^{15} \mathrm{O}_{6.176}^{*} \rightarrow \text { g.s. } \\
{ }^{15} \mathrm{~N}_{6.324}^{*} \rightarrow \text { g.s. }\end{array}$ & $180^{d}$ & $1.0 \times 10^{-5}$ \\
\hline
\end{tabular}

${ }^{a}$ For $E_{\text {esc }}=100 \mathrm{MeV}$ nucleon ${ }^{-1}$ and $\sigma=0.05 E_{\text {esc }}$ (see Eq. (4)). The fluxes are for the region of angular radius $\theta=5^{\circ}$ (see Fig. 3 for fluxes at 4.44 and $\sim 6.2 \mathrm{MeV}$ calculated for lower values of $\theta$ ). ${ }^{b}$ Ground state. ${ }^{c}$ Assuming thermal broadening with $T<10 \mathrm{keV}$. ${ }^{d}$ A very narrow line component at $6.129 \mathrm{MeV}$ could arise from excitation of ${ }^{16} \mathrm{O}$ nuclei contained in micrometer-sized grains (see text).

than $10^{4} \mathrm{yr}$ can produce very narrow lines in the GC environment. The most intense of these lines is expected to be that at $1.809 \mathrm{MeV}$ from the decay of ${ }^{26} \mathrm{Al}\left(T_{1 / 2}=7.2 \times 10^{5}\right.$ years $)$. This radioisotope can be synthesized by the reactions ${ }^{26} \mathrm{Mg}(\mathrm{p}, \mathrm{n}){ }^{26} \mathrm{Al}$ and ${ }^{28} \mathrm{Si}(\mathrm{p}, \mathrm{x}){ }^{26} \mathrm{Al}$ (Ramaty et al. 1979). However, the calculated flux in the $1.809 \mathrm{MeV}$ line is only $3.5 \times 10^{-7}$ photons $\mathrm{cm}^{-2} \mathrm{~s}^{-1}$ (Table 1). In comparison, the flux observed with INTEGRAL/SPI from the inner Galaxy $\left(-30^{\circ}<l<30^{\circ} ;-10^{\circ}<b<10^{\circ}\right)$ is $(3.3 \pm 0.4) \times 10^{-4} \mathrm{~cm}^{-2} \mathrm{~s}^{-1} \mathrm{rad}^{-1}$. It is produced by a presentday equilibrium mass of $2.8 \pm 0.8 M_{\odot}$ of ${ }^{26} \mathrm{Al}$ synthesized by massive stars in the Milky Way (Diehl et al. 2006).

The gamma-ray lines above $4 \mathrm{MeV}$ are better candidates for a future detection. Our predicted fluxes, however, are below currently available sensitivity limits of INTEGRAL. After one year of observation and $\sim 3 \mathrm{Ms}$ of exposure of the GC region, the SPI sensitivity for detection of a narrow line at $\sim 5 \mathrm{MeV}$ from a small-scale diffuse region at the center of the Galaxy was $3.2 \times 10^{-5} \mathrm{~cm}^{-2} \mathrm{~s}^{-1}$ (Teegarden \& Watanabe 2006, see Table 5). But for a broad line of $\sim 200 \mathrm{keV} \mathrm{FWHM}$ (see Table 1) the sensitivity limit increases by a factor of $\sim 8$. It is thus unlikely that the predicted gamma-ray line emission will be detected with INTEGRAL.

\section{Discussion and conclusions}

In spite of many uncertainties of the model parameters, we should state that their estimates cannot vary more than an order of magnitude. Thus, the injection energy should be close to $100 \mathrm{MeV}$, the average energy output is about $10^{41} \mathrm{erg} \mathrm{s}^{-1}$, and the spatial diffusion coefficient is about $10^{27} \mathrm{~cm}^{2} \mathrm{~s}^{-1}$ in order to reproduce the observed spatial distribution of hard X-rays in the GC (Dogiel et al. 2009a,b,c).

We showed that the accretion of stars onto the central supermassive black hole periodically ejects an intense flux of subrelativistic protons and nuclei capable of producing a significant emission of nuclear de-excitation gamma-ray lines in the 
GC region. The production rate of these energetic particles is about $10^{45} \mathrm{~s}^{-1}$. Because the time period of star capture by the black hole is expected to be shorter than the lifetime of the ejected protons against Coulomb losses, the density of these particles in the GC region is expected to be almost stationary. The radius of the volume filled with these energetic particles is found to be $\$ 700 \mathrm{pc}$ or $\$ 5^{\circ}$ if observed from Earth.

Based on the spectrum of the diffuse hard X-ray emission observed with Suzaku from the GC region (Yuasa et al. 2008), we estimated the energy of the nuclei accelerated in the vicinity of the black hole to be $E_{\mathrm{esc}} \approx 100 \mathrm{MeV}$ nucleon $^{-1}$. Further assuming that the mean metallicity in the GC region is two times higher than in the solar neighborhood (see, e.g., Koyama et al. 2009), we calculated the total gamma-ray line flux below $8 \mathrm{MeV}$ to be $1.1 \times 10^{-4}$ photons $\mathrm{cm}^{-2} \mathrm{~s}^{-1}$. The most promising lines for detection are those at 4.44 and $\sim 6.2 \mathrm{MeV}$, with a predicted flux in each line of $\approx 10^{-5}$ photons $\mathrm{cm}^{-2} \mathrm{~s}^{-1}$. These lines should be broad, $\Delta E_{\gamma} / E_{\gamma}$ of 3-4\%, which unfortunately renders their detection with the INTEGRAL spectrometer unlikely.

But future gamma-ray missions like the Nuclear Compton Telescope (Chang et al. 2007), the GRIPS project (Greiner et al. 2008), and the Advanced Compton Telescope (Boggs et al. 2006) may be able to test our predictions. In particular, the GRIPS mission proposed for ESA's "Cosmic Vision" program could achieve more than an order of magnitude sensitivity improvement after 5 years in orbit over COMPTEL (in 9 years), which would allow clear detection of the predicted gammaray line emission at 4.44 and $\sim 6.2 \mathrm{MeV}$ from the GC region. The Advanced Compton Telescope project proposed as a future NASA mission aims at even better sensitivity, near $10^{-6}$ photons $\mathrm{cm}^{-2} \mathrm{~s}^{-1}$ for $3 \%$ broad lines. A future detection of the predicted gamma-ray lines with such an instrument would provide unique information on the high-energy processes induced by the the central black hole, as well as on the physical conditions of the emitting region.

Finally, we note that nuclear interactions of subrelativistic ions with ambient material can also synthesize $\beta^{+}$radioisotopes, whose decay can inject positrons into the GC region. From the radioisotope production yields given by Kozlovsky et al. (1987), we estimate that, for $E_{\mathrm{esc}} \approx 100 \mathrm{MeV}$ nucleon $^{-1}$, the number of positrons produced by this mechanism is $\lesssim 5$ times the number of gamma-rays emitted in the 4.44 and $\sim 6.2 \mathrm{MeV}$ lines. With a predicted gamma-ray production rate of $1.7 \times 10^{41}$ photons s $^{-1}$ as the sum of these two lines (see Table 1), it gives a positron production rate $\lesssim 8.4 \times 10^{41} \beta^{+} \mathrm{s}^{-1}$. This limit is more than an order of magnitude lower than the positron annihilation rate measured with INTEGRAL/SPI (Weidenspointner et al. 2008).

Acknowledgements. First of all we would like to mention that the referee's report was very helpful for us and we thank him for comments. The authors are also grateful to H.-K. Chang, Y. Chou, Ya. N. Istomin, and Y. Maeda, for helpful discussions, and to W. Hermsen and $\mathrm{H}$. Bloemen for valuable input.

V.A.D. and D.O.C. are partly supported by the RFBR grant 08-02-00170-a, the NSC-RFBR Joint Research Project RP09N04 and 09-02-92000-HHC-a, and by the grant of the President of the Russian Federation "Scientific School of Academician V.L. Ginzburg". K.S.C. is supported by an RGC grant of the Hong Kong Government under HKU 7014/07P and a National Basic Research Program of China under 2009CB824800. C.M.K. is supported by the Taiwan National Science Council grants NSC 96-2112-M-008-014-MY3 and NSC-982923-M-008-001-MY3. W.H.I. is supported by the Taiwan National Science Council grants NSC 96-2752-M-008-011-PAE and NSC 96-2111-M-008-010.

The last version of the paper was partly prepared in ISAS/JAXA (Sagamihara, Japan). V.A.D. thanks ISAS and particularly Prof. Kazuhisa Mitsuda and the ISAS director Prof. Hajime Inoue for hospitality.

\section{References}

Abraham, J., Abreu, P., Aglietta, M. et al. (Pierre Auger Collaboration), 2007, Science, 318, 938

Alexander, T. 2005, PhR, 419, 65

Alexander, T., \& Livio, M. 2004, ApJ, 606, L21

Ayal, S., Livio, M., \& Piran, T. 2000, ApJ, 545, 772

Baganoff, F. K., Maeda, Y., Morris, M., et al. 2003, ApJ, 591, 891

Berezinskii, V. S., Bulanov, S. V., Dogiel, V. A., Ginzburg, V. L., \& Ptuskin, V. S. 1990, Astrophysics of Cosmic Rays, ed. V. L. Ginzburg (Amsterdam: Norht-Holland)

Bloemen, H., Bykov, A. M.., Diehl, R., et al. 1997, Proceedings of the Fourth Compton Symposium, ed. C. D. Dermer, M. S. Strickman, \& J. D. Kurfess, Williamsburg, VA, AIP Conf. Proc., 410, 107

Boggs, S., Kurfess, J., Ryan, J., et al. 2006, Space Telescopes and Instrumentation II: Ultraviolet to Gamma Ray, ed. M. J. L. Turner, \& G. Hasinger, Proc. SPIE, 6266, 62

Chang, H.-K., Boggs, S., \& Chang, Y.-H. 2007, Adv. Space Res., 40, 1281 Cheng, K. S., Chernyshov, D. O., \& Dogiel, V. A. 2006, ApJ, 645, 1138

Cheng, K. S., Chernyshov, D. O., \& Dogiel, V. A. 2007, A\&A, 473, 351

Cunha, K., Sellgren, K., Smith, V. V., et al. 2007, ApJ, 669, 1011

Diehl, R., Halloin, H., Kretschmer, K., et al. 2006, Nature, 439, 45

Dogiel, V. A. 2001, Proc. 4th Integral Workshop, in Exploring the Gamma-Ray Universe, ed. A. Gimenez, V. Reglero, \& C. Winkler, ESA SP-459, 139

Dogiel, V. A., Gurevich, A. V., Istomin, Ya. N., \& Zybin, K. P. 1987, MNRAS, 228,843

Dogiel, V. A., Gurevich, A. V., Istomin, Ya. N., \& Zybin, K. P. 2005, Ap\&SS, 297, 201

Dogiel, V. A., Cheng, K.-S., Chernyshov, D. O., et al. 2008, New Astron. Rev., 52,460

Dogiel, V., Chernyshov D., Cheng, K.-S., et al. 2009a, PASJ, 61, 901

Dogiel, V., Chernyshov D., Yuasa, T., et al. 2009b, to be published in PASJ, 61, No. 5

Dogiel, V., Chernyshov D., Yuasa, T., et al. 2009c, to be published in PASJ, 61, No. 5

Donley, J. L., Brandt, W. N., Eracleous, M., \& Boller, Th. 2002, ApJ, 124, 1308 Gillessen, S., Eisenhauer, F., Trippe, S., et al. 2008, A Giant Step: from Milli- to Micro-arcsecond Astrometry, Proc. IAU, IAU Symp., 248, 466 [arXiv: 0810.4674]

Gratton, L. 1972, Ap\&SS, 16, 81

Greiner, J., Iyudin, A., Kanbach, G., et al. 2008, Exper. Astron., 23, 91

Grönewegen, M. A. T., Udalski, A., \& Bono, G. 2008, A\&A, 481, 441

Hayakawa, S. 1969, Cosmic Ray Physics (Wiley-Interscience)

Istomin, Ya. N., \& Sol, H. 2009, Ap\&SS, 321, 57I

Iyudin, A. F., Böhringer, H., Dogiel, V. A., \& Morfill, G. 2004, A\&A, 413, 817

Jean, P., Knödlseder, J., Gillard, W., et al. 2006, A\&A, 445, 579

Kiener, J., Gros, M., Tatischeff, V., \& Weidenspointner, G. 2006, A\&A, 445, 725

Koyama, K., Maeda, Y., Sonobe, T., et al. 1996, PASJ, 48, 249

Koyama, K., Hyodo, Y., Inui, T., et al. 2007, PASJ, 59, 245

Koyama, K., Takikawa, Y., Hyodo, Y., et al. 2009, PASJ, 61, S255

Kozlovsky, B., Lingenfelter, R. E., \& Ramaty, R. 1987, ApJ, 316, 801

Kozlovsky, B., Murphy, R. J., \& Ramaty, R. 2002, ApJS, 141, 523

LaRosa, T. N., Lazio, T., Joseph, W., \& Kassim, N. E. 2001, ApJL, 563, 163

Launhardt, R., Zylka, R., \& Mezger, P. G. 2002, A\&A, 384, 112

Lingenfelter, R. E., \& Ramaty, R. 1977, ApJ, 211, L19

Lodders, K. 2003, ApJ, 591, 1220

Maeda, Y., Baganoff, F. K., Feigelson, E. D., et al. 2002, ApJ, 570, 671

Morris, M., \& Serabyn, E. 1996, ARA\&A, 34, 645

Muno, M. P., Baganoff, F. K., Bautz, M. W., et al. 2004, ApJ, 613, 326

Phinney, E. S. 1989, Nature, 340, 595

Porquet, D., Grosso, N., Predehl, P., et al. 2008, A\&A, 488, 549

Ramaty, R., Kozlovsky, B., \& Lingenfelter, R. E. 1979, ApJS, 40, 487

Rees, M. J. 1988, Nature, 333, 523

Sirota, V. A., Il'in, A. S., Zybin, K. P., \& Gurevich, A. V. 2005, JETP, 100, 294

Skilling, J., \& Strong, A. W. 1976, A\&A, 53, 253

Smith, D. M., Share, G. H., Murphy, R. J. et al. 2003, ApJ, 595, L81

Syer, D., \& Ulmer, A. 1999, MNRAS, 306, 35

Syrovatskii, S. I. 1959, Sov. Astron., 3, 22

Tatischeff, V., \& Kiener, J. 2004, New Astron. Rev., 48, 99

Teegarden, B. J., \& Watanabe, K. 2006, ApJ, 646, 965

Ulmer, A. 1999, ApJ, 514, 180

Weidenspointner, G., Skinner, G., Jean, P., et al. 2008, Nature, 451, 159

Yuasa, T., Tamura, K., Nakazawa, K., et al. 2008, PASJ, 60, S207 